\title{
时间变换过程的 Revuz 测度
}

何 萍 $^{1}$ 应坚刚 ${ }^{2}$

(1. 上海财经大学应用数学系, 上海 $200433 ; 2$. 复旦大学数学研究所, 上海 200433)

摘要本文证明 Markov 过程的能量泛函与 Revuz 测度在时间变换下保持不变, 并通过直 接计算的方法导出时间变换过程的 Lévy 系统和跳跃测度.

关键词 时间变换 连续加泛函 跳跃测度 Feller 测度

$\operatorname{MSC}(2000)$ 主题分类 $60 \mathrm{~J} 45,60 \mathrm{~J} 40$

\section{1 前言}

时间变换是 Markov 过程论的重要变换之一. 本文研究状态空间 $(E, \mathscr{E})$ 上的右 Markov 过程 (其定义可参见文献 [1])

$$
X=\left(\Omega, \mathscr{F}, \mathscr{F}_{t}, X_{t}, \theta_{t}, \mathbb{P}^{x}\right),
$$

相应转移半群为 $\left(P_{t}\right), \partial$ 为坟墓状态, $\zeta$ 为过程的生命时间. 为方便起见, 有时也将 $X_{t}$ 写 成 $X(t)$. 如果一个非负的右连续过程 $A=\left(A_{t}\right)$ 满足 $A_{0}=0$ 并且对任意 $s, t \geqslant 0, A_{t+s}=$ $A_{t}+A_{s} \circ \theta_{t}$ 几乎处处成立, 则称 $A$ 为加泛函. 在这里, 我们所提到的加泛函都是适应的. 如果 关心加泛函的相关性质, 可以参见文献 [2]. 给定一个连续的加泛函 $A$, 定义

$$
\begin{aligned}
& \tau_{t}:=\inf \left\{s>0: A_{s}>t\right\}, \\
& R_{A}:=\inf \left\{t>0: A_{t}>0\right\}, \\
& F:=\left\{x: \mathbb{P}^{x}\left(R_{A}=0\right)=1\right\}, \\
& T:=\inf \left\{t>0: X_{t} \in F\right\},
\end{aligned}
$$

这里, $\left(\tau_{t}\right)$ 是 $A$ 的右连续逆, $F$ 是 $A$ 的精细支撑. $\tau_{0}=R_{A}=T$ (参见文献 [1], §64). 记 $\Phi(x):=\mathbb{E}^{x}\left(e^{-T}\right), \mathscr{E} e$ 是由 $X$ 的 1- 过分函数生成的 $\sigma-$ 代数, 有 $F=\{\Phi=1\} \in \mathscr{E}^{e}$. 显然, $\left\{\tau_{t}: t \geqslant 0\right\}$ 是一个递增的停时列并且 $\tau_{0}=R_{A}$. 记 $\tilde{X}_{t}=X_{\tau_{t}}, \tilde{\mathscr{F}}_{t}=\mathscr{F}_{\tau_{t}}, \tilde{\theta}_{t}=\theta_{\tau_{t}}$, 则过程

$$
\tilde{X}=\left(\Omega, \mathscr{F}, \tilde{\mathscr{F}}_{t}, \tilde{X}_{t}, \tilde{\theta}_{t}, \mathbb{P}^{x}\right)
$$

是以 $F$ 为状态空间, 生命时间为 $\tilde{\zeta}=A_{\infty}$ 的右 Markov 过程. 称 $\tilde{\theta}$ 为 $X$ 关于 $A$ 的时间变换 过程, 该过程与原过程的一些谱性质保持一致. 比如, 首中分布在过程的时间变换下就保持不 变. 关于时间变换的符号与结论请参照文献 [1]. 本文将证明能量泛函和 Revuz 测度在时间 
变换下保持不变, 并推导时间变换过程的 Lévy 系统和跳跃测度. 本文的研究动机源于早期的 论文 ${ }^{[3-5]}$ 中关于 Douglas 积分的论题, 他们的工作探讨了在一个有界区域上的经典 Dirichlet 积分留在边界上的迹. 而近期的论文 ${ }^{[6,7]}$ 在假设 $X$ 关于测度 $m$ 持弱对偶性并且半 - 极集是 $m$ - 极集的前提下, 推导出时间变换过程跳跃测度的具体表达. 本文更深入地探讨时间变换过 程的谱理论, 并采用更加直观的方法证明了文献 [6] 中的一个主要结果.

\section{2 时间变换过程的 Revuz 测度}

本节假设 $X$ 为状态空间 $(E, \mathscr{E})$ 是 Radon 空间的右过程. 对 $E$ 上所有函数取 $f(\partial)=0$ 延拓到 $E \cup\{\partial\}$. 设 $A$ 是 $X$ 的以精细完美集合 $F$ 为精细支撑的连续加泛函. 设 $\tau=\left(\tau_{t}\right)$ 为 $A$ 的右连续逆, $\tau$ 是递增的并且当且仅当 $A$ 严格递增时连续. 记 $\tilde{X}$ 为上节所述的时间变换 过程, 亦称为 $X$ 在 $F$ 上的迹. 令

$$
M:=\overline{\left\{t: X_{t} \in F\right\}},
$$

这是一个齐性的随机闭集. 直观地, 称构成余集 $M^{c}$ 的开区间为 $F$ 上的游离区间. 设 $T$ 为 $F$ 的首中时, 则 $A_{\tau_{t}}=t, R_{A}=T$ 并且 $\tau_{A_{t}}=T \circ \theta_{t}+t$, 从而

$$
M=\overline{\left\{T \circ \theta_{t}+t: t>0\right\}}=\overline{\left\{\tau_{t}: t>0\right\}},
$$

只要将 $F$ 上的游离部分抹去就可以得到 $\tilde{X}$. 这正是我们把 $\tilde{X}$ 称为 $X$ 留在 $F$ 上的迹的理由. 一个连续加泛函 $A$ 以 $F$ 为精细支撑, 意味着测度 $d A_{t}$ 是以 $M$ 为支撑的. 由于

$$
\tau_{s} \circ \theta_{\tau_{t}}+\tau_{t}=\tau_{t+s},
$$

如果 $H$ 是 $X$ 的另一加泛函, 那么 $\tilde{H}:=\left(H_{\tau_{t}}\right)$ 是 $\tilde{X}$ 的加泛函. 称加泛函 $H=\left(H_{t}\right)$ 是以 $F$ 为 支撑的, 如果对于任意 $t, H_{t}=\int_{0}^{t} 1_{F}\left(X_{s}\right) d H_{s}$, 换句话说, $H$ 离开 $F$ 就不递增, 其精细支撑包 含于 $F$. 显然, $H$ 以 $F$ 为支撑的充要条件是 $H$ 是 $\tau$ - 连续的, 即对任意 $t \geqslant 0, t \mapsto B_{t}$ 在区间 $\left[\tau_{t-}, \tau_{t}\right]$ 上是一个常数.

假设 $A$ 严格递增, 也就是在 $E$ 上有精细支撑. 此时, $A$ 的逆连续并且其时间变换可逆, $X$ 即为 $\tilde{X}$ 的时间变换, 从而 $X$ 与 $\tilde{X}$ 互为时间变换. 著名的 Blumenthal-Getoor-McKean 定理 指出, 两个 Markov 过程关于严格递增加泛函互为时间变换的充要条件是它们有相同的首中 分布. 所以, 关于严格递增的连续加泛函的时间变换针对 $E$ 上右过程的全体是一个等价关系.

引理 2.1 如果一个连续加泛函 $B$ 以 $F$ 为支撑, 则 $t \mapsto B_{\tau_{t}}$ 是连续的. 如果两个连续加 泛函 $A$ 与 $B$ 有相同的精细支撑, 则它们各自对应的时间变换 $\tilde{X}$ 与 $\tilde{X}^{\prime}$ 关于某个严格递增的 连续加泛函互为时间变换.

证明 第 1 个结论的证明是显然的. 我们仅证明第 2 个结论. 由于 $B$ 也是以 $F$ 为支撑 集的, 设 $\sigma$ 是 $B$ 的逆, 则 $B_{\tau}$ 是 $\tilde{X}$ 的一个递增的连续加泛函, 它的逆为 $A_{\sigma}$. 所以

$$
\tilde{X}\left(A_{\sigma}\right)=X(\sigma)=\tilde{X}^{\prime} .
$$

给定上述 $X$ 及其关于连续加泛函 $A$ 的、以 $F$ 为精细支撑的时间变换过程 $\tilde{X}$. 设 $U^{\alpha}$ 和 $\tilde{U}^{\alpha}$ 分别为 $X$ 与 $\tilde{X}$ 的 $\alpha$ - 预解. 令 $P_{F}^{\alpha}$ 为 $F$ 的 $\alpha$ - 首中核,

$$
P_{F}^{\alpha}(x, d y)=\mathbb{E}^{x}\left(e^{-\alpha T} ; X_{T} \in d y\right),
$$

这是一个定义在 $E \times \mathscr{B}(F)$ 上的核. 当 $\alpha$ 取值为 0 时, 记 $P_{F}^{0} \equiv P_{F}$. 对任意加泛函 $H, U_{H}^{\alpha}$ 是 $H$ 的 $\alpha$ - 谱. 下面的基本结果在全文中很有用. 设 $f, g, \tau$ 为 3 个 $[0, \infty)$ 上的非负右连续函数. 
如果 $\tau$ 与 $g$ 都递增并且 $g$ 是 $\tau$ - 连续的, 则有如下变量变换公式:

$$
\int_{0}^{\infty} f(s) d g(s)=\int_{0}^{\infty} f(\tau(s)) d g(\tau(s)) .
$$

引理 $2.2 U_{A}=P_{F} \tilde{U}$.

证明 因为对于 $t \leqslant T, A_{t}=0$. 由强 Markov 性,

$$
\begin{aligned}
U_{A} f(x) & =\mathbb{E}^{x} \int_{0}^{\infty} f\left(X_{t}\right) d A_{t}=\mathbb{E}^{x}\left(\int_{T}^{\infty} f\left(X_{t}\right) d A_{t} ; T<\infty\right) \\
& =\mathbb{E}^{x}\left(\int_{0}^{\infty} f\left(X_{t+T}\right) d A_{t+T} ; T<\infty\right)=\mathbb{E}^{x}\left(\mathbb{E}^{X_{T}} \int_{0}^{\infty} f\left(X_{t}\right) d A_{t} ; T<\infty\right) \\
& =\mathbb{E}^{x}\left(\mathbb{E}^{X_{T}} \int_{0}^{\infty} f\left(X_{\tau_{t}}\right) d t ; T<\infty\right)=P_{F} \tilde{U} .
\end{aligned}
$$

引理 2.3 如果 $E$ 上的一个函数 $h$ 关于 $X$ 过分, 那么 $\left.h\right|_{F}$ 关于 $\tilde{X}$ 也是过分的. 反之, 如果 $F$ 上的函数 $h$ 关于 $\tilde{X}$ 过分, 那么 $P_{F} h$ 关于 $X$ 过分.

证明 第 1 个结论是文献 [1] 中的练习. 仅证引理的后半部. 设 $S^{\alpha}(X)$ 是 $X$ 的过分 函数的全体, $S(X):=S^{0}(X)$, 则 $S(X)=\bigcap_{\alpha>0} S^{\alpha}(X)$. 我们只需证明 $h \in S^{\alpha}(\tilde{X})$ 隐含着 $P_{F} h \in S^{\alpha}(X)$ 对 $\alpha>0$ 成立, 或更强地, 在 $\tilde{X}$ 是暂留的条件下证明第 2 个结论. 而 $X$ 暂留意 味着 $h$ 是递增谱序列 $\tilde{U} f_{n}$ 的极限. 由上一个引理可得 $U_{A} f_{n}=P_{F} \tilde{U} f_{n}$, 从而 $U_{A} f_{n} \uparrow P_{F} h$, 即 $P_{F} h$ 是 $S(X)$ 中的过分函数序列的极限, 所以 $P_{F} h \in S(X)$ 成立.

该引理告诉我们, 对于 $F$ 上任意函数 $h, h \in S(\tilde{X})$ 等价于 $P_{F} h \in S(X)$, 所以 $X$ 有不平 凡的过分函数的充要条件是 $\tilde{X}$ 有不平凡的过分函数. 而 $X$ 常返的充要条件是 $X$ 的所有过 分函数恒为常数, 从而 $X$ 常返当且仅当 $\tilde{X}$ 常返, 过程的时间变换不改变其常返性.

设 $\operatorname{Exc}(X)$ 为 $X$ 所有过分函数的全体, $L$ 与 $\tilde{L}$ 分别是 $X$ 和 $\tilde{X}$ 的能量泛函. 给定 $m \in$ $\operatorname{Exc}(X)$ 与加泛函 $H$, 定义 $H$ 关于 $m$ 的 Revuz 测度 $\xi_{H}^{m}$ 如下: 对 $f \in \mathscr{E}_{+}$,

$$
\xi_{H}^{m}(f):=\lim _{t \downarrow 0} \frac{1}{t} \mathbb{E}^{m} \int_{0}^{t} f\left(X_{t}\right) d H_{t},
$$

这是一个递增的极限. 应该注意该测度也与过程 $X$ 有关, 如果有必要我们会声明是针对 $X$ 进行计算的. 显然 $\xi_{H}^{m}$ 的支撑是 $H$ 的精细支撑. 不难证明 Revuz 测度 $\xi_{A}^{m}=: \tilde{m}$ 是 $\tilde{X}$ 的过分 测度, 即 $\tilde{m} \in \operatorname{Exc}(\tilde{X})$. 下面讨论能量泛函的关系.

定理 2.1 设 $m \in \operatorname{Exc}(X), \tilde{m}$ 为上述 Revuz 测度. 如果 $m$ 是耗散的, 则 $\tilde{m}$ 也是耗散的, 并且对于任意 $h \in S(\tilde{X})$, 有

$$
L\left(m, P_{F} h\right)=\tilde{L}(\tilde{m}, h) .
$$

证明 存在一个谱序列 $\eta_{n} U$, 使得 $\eta_{n} U \uparrow m$, 从而 $\eta_{n} U_{A} \uparrow \tilde{m}$ (参见文献 [8]). 而 $U_{A}=$ $P_{F} \tilde{U}$, 所以 $\left(\eta_{n} P_{F}\right) \tilde{U} \uparrow \tilde{m}$, 可知 $\tilde{m}$ 关于 $\tilde{X}$ 耗散. 我们有

$$
L\left(m, P_{F} h\right)=\uparrow \lim \eta_{n} P_{F}(h)=\tilde{L}(\tilde{m}, h) .
$$

该定理描述了过程 $X$ 与 $\tilde{X}$ 谱理论之间的关系. 比如考虑容度. 参照文献 [8] 固定 $m \in$ $\operatorname{Exc}(X)$, 对于 $B \subset E$ 且 $B \in \mathscr{E} e$, 定义 $B$ 的容度为 $\Gamma(B):=L\left(m, P_{B} 1\right)$, 称 $\Gamma$ 为 $X$ 关于 $m$ 的容度. 设 $\Gamma$ 和 $\tilde{\Gamma}$ 分别为 $X$ 关于 $m$ 与 $\tilde{X}$ 关于 $\tilde{m}$ 的容度. 设 $B \subset F$ 且 $B \in \mathscr{E}^{e}$, 显然 $\left\{T_{B}<\infty\right\}=\left\{\tilde{T}_{B}<\infty\right\}$ 并且在 $\left\{T_{B}<\infty\right\}$ 上, $X\left(T_{B}\right)=\tilde{X}\left(\tilde{T}_{B}\right)$. 所以对于任意的 $x \in F$,

$$
\tilde{P}_{B} f(x)=\mathbb{E}^{x}\left(f\left(\tilde{X}\left(\tilde{T}_{B}\right)\right) ; \tilde{T}_{B}<\infty\right)=P_{B} f(x),
$$


并且

$$
\tilde{\Gamma}(B)=\tilde{L}\left(\tilde{m}, \tilde{P}_{B} 1\right)=L\left(m, P_{F} P_{B} 1\right)=L\left(m, P_{B} 1\right)=\Gamma(B) .
$$

这意味着时间变换不改变 $F$ 的任何一个子集的容度.

如果 $A$ 是一个递增, 那么 $S(X)=S(\tilde{X})$ 并且对于 $m \in S(X), L(m, h)=L(\tilde{m}, h)$. 下叙定 理说明在一定的前提下, 时间变换不改变 Revuz 测度. 设 $H$ 是 $X$ 的一个加泛函, $\tilde{H}_{t}:=H_{\tau_{t}}$, 则 $\tilde{H}$ 是 $\tilde{X}$ 的一个加泛函. 若 $H$ 连续并以 $F$ 为支撑, 则 $\tilde{H}$ 也连续. 定义 $\tilde{\xi}_{\tilde{H}}^{\tilde{m}}$ 为 $\tilde{X}$ 下 $\tilde{H}$ 关于 $\tilde{m}$ 的 Revuz 测度, 称之为由 $A$ 诱导的 $H$ 的 Revuz 测度.

定理 2.2 如果 $H$ 是以 $F$ 为支撑的加泛函, 那么

$$
\xi_{H}^{m}=\tilde{\xi}_{\tilde{H}}^{\tilde{m}} .
$$

证明 当 $m$ 耗散时, 结论显然成立. 由于 $H$ 的支撑集是 $F$, 对于 $t<T$ 有 $H_{t}=0$. 根据 强 Markov 性, 有

$$
U_{H} f(x)=\mathbb{E}^{x} \int_{T}^{\infty} f\left(X_{t}\right) d H_{t}=P_{F} \tilde{U}_{\tilde{H}} f(x),
$$

即 $U_{H}=P_{F} \tilde{U}_{\tilde{H}}$. 由定理 2.1 可知

$$
\xi_{H}^{m}(f)=L\left(m, U_{H} f\right)=L\left(m, P_{F} \tilde{U}_{\tilde{H}} f\right)=\tilde{L}\left(\tilde{m}, \tilde{U}_{\tilde{H}} f\right)=\tilde{\xi}_{\tilde{H}}^{\tilde{m}}(f) .
$$

对于一般的场合, 需要考虑 $X$ 的 1- 子过程 $X^{1}$, 因为 $m$ 关于 $X^{1}$ 是耗散的. 在 $X^{1}$ 之下, 仍记 $H$ 关于 $m$ 的 Revuz 测度为 $\xi_{H}^{m}$. 类似于引理 2.2 的证明, 有

$$
\begin{aligned}
& U_{A}^{1}=P_{F}^{1} \tilde{U}_{M * \tilde{A}}, \\
& U_{H}^{1}=P_{F}^{1} \tilde{U}_{M * \tilde{H}},
\end{aligned}
$$

其中 $M_{t}=e^{-\tau_{t}}$ 是 $\tilde{X}$ 的乘积泛函, $d M * \tilde{A}=M_{t} d \tilde{A}_{t}, d M * \tilde{H}=M_{t} d \tilde{H}_{t}$. 注意到 $\tilde{A}_{t}=t$, 即 $\tilde{U}_{M * \tilde{A}}$ 是 $\tilde{X}$ 被 $M$ killing 的子过程的谱 (即 0- 预解), 所以 $X^{1}$ 关于 $A$ 的时间变换过程等价于 $\tilde{X}$ 被 $M$ 切断的 killing 过程 (这里所采用的符号可参照文献 [9].) 由于 $H$ 是 $\tau$ - 连续的, 所以 $\tau$ 和 $\tilde{H}$ 没有公共的不连续点, 并且 $M * \tilde{H}=M_{-} * \tilde{H}$. 下面, 取一单调递增收玫于 $m$ 的谱序 列 $\left\{\eta_{n} U^{1}\right\}$, 有

$$
\eta_{n} P_{F}^{1} \tilde{U}_{M * \tilde{H}}=\eta_{n} U_{H}^{1} \uparrow \xi_{H}^{m} .
$$

另一方面, $\eta_{n} U_{A}^{1} \uparrow \tilde{m}$, 即 $\eta_{n} P_{F}^{1} \tilde{U}_{M * \tilde{A}} \uparrow \tilde{m}$. 由定理 2.4 与文献 [9] 的命题 2.3(d), 有

$$
\tilde{\xi}_{\tilde{H}}^{\tilde{m}}=\tilde{\xi}_{M_{-} * \tilde{H}}^{\tilde{m}}=\lim _{n} \eta_{n} P_{F}^{1} \tilde{U}_{M_{-} * \tilde{H}}=\xi_{H}^{m} .
$$

定理得证.

该定理告诉我们, 由任意连续加泛函诱导的持有相同支撑的关于 $H$ 的测度都是相同的.

推论 2.1 如果 $A$ 严格递增, 那么对任意加泛函 $H$,

$$
\xi_{H}^{m}=\tilde{\xi}_{\tilde{H}}^{\tilde{m}} .
$$

\section{3 时间变换过程的 Lévy 系统与跳跃测度}

假设 $X$ 是一个标准 Markov 过程以确保其 Lévy 系统存在 (参见文献 [1], 说明更弱的条 件也可保证这种存在性; 本文并不需要更一般的条件). 设 $N$ 是 $E \times \mathscr{E}$ 上满足 $N(x,\{x\})=0$ 
对任意 $x \in E$ 成立的核, $H$ 是 $X$ 的连续加泛函. 称偶 $(N, H)$ 为 $X$ 的 Lévy 系统, 如果对任 意正的可预测过程 $Z=\left(Z_{t}\right)$ 和 $E \times E_{\partial}$ 上在对角线消失的正可测函数 $f$,

$$
\mathbb{E}^{x} \sum Z_{t} f\left(X_{t-}, X_{t}\right)=\mathbb{E}^{x} \int_{0}^{\infty} Z_{t} \int_{E_{\partial}} N\left(X_{t}, d y\right) f\left(X_{t}, y\right) d H_{t}
$$

对于任意 $x \in E$ 都成立. 事实上, 我们只需用到 $Z_{t}=e^{-t}$ 的场合. 记

$$
N f=\int_{E_{\partial}} N(\cdot, d y) f(\cdot, y) .
$$

我们通常以下叙意义表示 Lévy 的唯一性. 设 $X$ 的两个 Lévy 系统分别为 $\left(N^{1}, H^{1}\right)$ 与 $\left(N^{2}, H^{2}\right)$, 如果对任意上述 $f$ 有

$$
\int_{0}^{t} N^{1} f\left(X_{s}\right) d H_{s}^{1}=\int_{0}^{t} N^{2}\left(X_{s}\right) d H_{s}^{2}
$$

几乎处处成立, 那么该过程的 Lévy 系统是唯一的. Lévy 系统刻画 $X$ 在 $E$ 中是如何跳跃并如 何跳进坟墓. 如果

$$
\begin{aligned}
\mathbb{E}^{x} \sum Z_{t} f\left(X_{t-}, X_{t}\right)= & \mathbb{E}^{x} \int_{0}^{\infty} Z_{t} \int_{E_{\partial}} N\left(X_{t}, d y\right) f\left(X_{t}, y\right) d H_{t} \\
& +\mathbb{E}^{x} \int_{0}^{\infty} Z_{t} \int_{E_{\partial}} N^{\prime}\left(X_{t}, d y\right) f\left(X_{t}, y\right) d H_{t}^{\prime},
\end{aligned}
$$

我们可以用一对系统 $(N, H)$ 与 $\left(N^{\prime}, H^{\prime}\right)$ 定义 Lévy 系统. 为更加简单直观, 常常把两个系统 合并成一个进行讨论. 对于 $m \in \operatorname{Exc}(X)$, 定义

$$
\begin{aligned}
& J(f)=\lim _{t \rightarrow 0} \frac{1}{t} \mathbb{E}^{m} \sum_{s \leqslant t} f\left(X_{s-}, X_{s}\right), \\
& k(g)=\lim _{t \rightarrow 0} \frac{1}{t} \mathbb{E}^{m}\left(g\left(X_{\zeta-}\right) ; \zeta \leqslant t\right)
\end{aligned}
$$

分别为 $X$ 的跳跃测度与 killing 测度, 其中 $f \geqslant 0$ 是 $E \times E$ 上在对角线附近消失的可测函数, $g \geqslant 0$ 是 $E$ 上的可测函数, 这两个测度实际上均为加泛函的 Revuz 测度, 所以当 $t \downarrow 0$ 时它们 各为递增序列的极限. 利用 Lévy 系统易证

$$
J(d x, d y)=N(x, d y) \xi_{H}^{m}(d x), \quad k(d x)=N(x, \partial) \xi_{H}^{m}(d x) .
$$

记 $\mathbf{S}_{F}$ 是 $X$ 的以 $F$ 为精细支撑集的连续加泛函的全体. 如果 $F$ 是闭的并且精细完美, 那么 $\mathbf{S}_{F}$ 为非空集. 对于 $\mathbf{S}_{F}$ 中一个固定的加泛函 $A$, 类似于 $\S 2$ 中由 $A$ 诱导 $X$ 的时间变换. 我们下面的目标在于 $\tilde{X}$ 的 Lévy 系统以及相应跳跃, killing 测度的计算. 直观上, $\tilde{X}$ 的跳跃 不仅会在 $F$ 中出现, 而且还由 $F$ 上所谓的 “游离” 诱导, 所以还需要引入流出系统.

为描述一个集合的游离, Maisoneuve ${ }^{[10]}$ 提出了流出系统, 这个概念的起源可以追溯到 Getoor-Sharpe $^{[11]}$ 或更早地 Motoo ${ }^{[12]}$ 的工作. 同于上节, 记 $M=\overline{\left\{t: X_{t} \in F\right\}}$. 令 $T_{t}=$ $T \circ \theta_{t}+t$

$$
G:=\left\{t>0: T_{t}>T_{t-}=t\right\},
$$

即集合 $M$ 在 $(0, \infty)$ 中所有左端点的全体. 记

$$
G^{i}:=\left\{t \in G: X_{t} \notin F^{r}\right\}, \quad G^{r}:=\left\{t \in G: X_{t} \in F^{r}\right\} .
$$

设 $\left(\Omega, \mathscr{F}^{0}\right)$ 到 $(E, \mathscr{E})$ 上的核 $\hat{\mathbb{P}}$ 对任意 $x$ 满足

$$
\hat{\mathbb{P}}^{x}(R=0)=0, \quad \hat{\mathbb{P}}^{x}\left(1-e^{-R}\right) \leqslant 1,
$$


并且对 $x \in E_{0}=E \backslash F, \hat{\mathbb{P}}^{x}=\mathbb{P}^{x}$. 所谓流出系统, 就是由上述核 $\hat{\mathbb{P}}$ 与 $X$ 正的加泛函 $L$ 构成的 一个偶 $(\hat{\mathbb{P}}, L)$, 使得对于 $\Omega$ 上任意正的随机变量 $f$ 和正过程 $Z=\left(Z_{t}\right)$, 有

$$
\mathbb{E}^{x} \sum_{s \in G} Z_{s} \cdot f \circ \theta_{s}=\mathbb{E}^{x} \int_{0}^{\infty} Z_{s} \hat{\mathbb{P}}^{X_{s}}(f) d L_{s}
$$

对于任意 $x \in E$ 成立.

文献 [10] 中证明了流出系统的存在性, 其中 $L=K+J, K$ 为 $F^{r}$ 上一个连续加泛函,

$$
d J_{t}=\sum_{s \in G^{i}} \varepsilon_{s}(d t) .
$$

如果对于任意正的可预测过程 $Z,(3.2)$ 式关于某可预测加泛函 $L$ 成立, 则称偶 $(\hat{\mathbb{P}}, L)$ 为 流出系统的可预测形式. 进一步地, 如果该可预测形式中的加泛函 $L$ 连续并以 $F$ 为支撑集, 则称之为连续加泛函构成的流出系统的可预测形式. 下面假设我们的流出系统可预测形式是 由 $F$ 上的连续加泛函所诱导的. 这里对什么条件下才能形成这种形式不作论述. 无论如何, 至少在 $F$ 为闭集并精细完美的场合结论是成立的 (参见文献 [13], 第 VII 章). 当 $F$ 为精细闭 集, $X$ 与另一个标准过程关于测度 $m$ 弱对偶, 并且 Hunt 假设 $(\mathrm{H})$ : 半 - 极集是 $m$ - 极的 ${ }^{[6]}$ 被 满足时, 上述结论也是成立的. 从现在开始, 我们可以通过计算推导 $\tilde{X}$ 的 Lévy 系统.

定理 3.1 系统 $(\tilde{N}, \tilde{K})$ 与 $(U, \tilde{L})$ 构成 $\tilde{X}$ 的 Lévy 系统.

证明 注意到 $G=\left\{\tau_{s-}: \tau_{s-}<\tau_{s}, s>0\right\}$, 不难证明 $\tau_{s-}=\tau_{s}$ 当且尽当 $X_{\tau_{s}} \in F$, $X_{\tau_{s}-} \in F$. 选取 $F \times F_{\partial}$ 上正的在对角线上消失的可测函数 $f$, 采用文献 $[6,7,14]$ 中所采用 的方法, 可得

$$
\begin{aligned}
\mathbb{E}^{x} \sum & e^{-s} f\left(\tilde{X}_{s-}, \tilde{X}_{s}\right) \\
& =\mathbb{E}^{x}\left(\sum e^{-s} f\left(X_{\tau_{s-}}, X_{\tau_{s}}\right)\right) \\
& =\mathbb{E}^{x}\left(\sum_{\tau_{s-}=\tau_{s}} e^{-s} f\left(X_{\tau_{s}-}, X_{\tau_{s}}\right)\right) 1_{F}\left(X_{\tau_{s}}\right)+\mathbb{E}^{x}\left(\sum_{\tau_{s-}<\tau_{s}} e^{-s} f\left(X_{\tau_{s-}}, X_{\tau_{s}}\right)\right) \\
& =\mathbb{E}^{x}\left(\sum e^{-A_{s}} f\left(X_{s-}, X_{s}\right)\right) 1_{F}\left(X_{s}\right)+\mathbb{E}^{x}\left(\sum_{s \in G} e^{-A_{s}} f\left(X_{s-}, X_{T} \circ \theta_{s}\right)\right) .
\end{aligned}
$$

因为 $A$ 连续并且 $f\left(X_{s-}, \cdot\right)$ 可预测, 利用 Lévy 系统和流出系统, 有

$$
\begin{aligned}
\mathbb{E}^{x} \sum e^{-s} f\left(\tilde{X}_{s-}, \tilde{X}_{s}\right)= & \mathbb{E}^{x} \int_{0}^{\infty} e^{-A_{s}} d H_{s} \int_{F \cup\{\partial\}} N\left(X_{s}, d y\right) f\left(X_{s}, y\right) 1_{F}(y) \\
& +\left.\mathbb{E}^{x} \int_{0}^{\infty} e^{-A_{s} \hat{\mathbb{P}}^{x}}\left(f\left(x, X_{T}\right)\right)\right|_{x=X_{s}} d L_{s} .
\end{aligned}
$$

记

$$
\tilde{N}(x, d y)=1_{F}(x) N(x, d y) 1_{F}(y), \quad K_{t}=1_{F}\left(X_{t}\right) d H_{t},
$$

并对 $x \in F, y \in F_{\partial}$, 记 $U(x, d y)=\hat{\mathbb{P}}^{x}\left(X_{T} \in d y\right)$. 由于 $K$ 与 $L$ 均为支撑在 $F$ 上的连续加泛 函, 它们均 $\tau$ - 连续, 从而 $\tilde{K}=K_{\tau}$ 与 $\tilde{L}=L_{\tau}$ 是 $\tilde{X}$ 的连续加泛函, 我们有

$$
\begin{aligned}
\mathbb{E}^{x} \sum e^{-s} f\left(\tilde{X}_{s-}, \tilde{X}_{s}\right) & =\mathbb{E}^{x} \int_{0}^{\infty} e^{-A_{s}} \tilde{N} f\left(X_{s}\right) d K_{s}+\mathbb{E}^{x} \int_{0}^{\infty} e^{-A_{s}} U f\left(X_{t}\right) d L_{s} \\
& =\mathbb{E}^{x} \int_{0}^{\infty} e^{-s} \tilde{N} f\left(\tilde{X}_{s}\right) d \tilde{K}_{s}+\mathbb{E}^{x} \int_{0}^{\infty} e^{-s} U f\left(\tilde{X}_{t}\right) d \tilde{L}_{s} .
\end{aligned}
$$


定理得证.

正如我们在证明过程中所提到过的，我们采用的证明方法与文献 $[3,7]$ 中采用的方法相 同. 直到最近我们才发现这种方法早在 1965 年 Motoo ${ }^{[12]}$ 的工作中已经出现过, 尽管当时只 证明一个关于 $\tilde{X}$ 的 Lévy 系统可以由系统 $(U, \tilde{L})$ 所控制.

综上所述, Lévy 系统似乎依赖于我们所选取的连续加泛函 $A$. 我们将推导出跳跃测度和 killing 测度的表达式同时证明它们是与 $A$ 无关的. 下叙定理在文献 [6] 中与本文不同的前提 下得到证明. 我们知道跳跃测度和 killing 测度的定义是针对某一个过分测度而言. 如果采取 $X$ 相应的测度 $m$, 那么针对 $\tilde{X}, \tilde{m}$ 是一个自然的选择. 记 $\tilde{J}$ 与 $\tilde{k}$ 分别为 $\tilde{X}$ 关于 $\tilde{m}$ 的跳跃测 度和 killing 测度. 易证

$$
\tilde{J}(d x, d y)=\tilde{N}(x, d y) \tilde{\xi}_{\tilde{K}}^{\tilde{m}}(d x)+U(x, d y) 1_{F}(y) \tilde{\xi}_{\tilde{L}}^{\tilde{m}}(d x) .
$$

由定理 2.2 可知

$$
\tilde{J}(d x, d y)=\tilde{N}(x, d y) \xi_{K}^{m}(d x)+U(x, d y) 1_{F}(y) \xi_{L}^{m}(d x) .
$$

因为 $\xi_{K}^{m}=1_{F} \cdot \xi_{H}^{m}$, 左式实际是 $J$ 在 $F \times F$ 上的限制, 记为 $J_{F}$. 记

$$
U(d x, d y)=\xi_{L}^{m}(d x) U(x, d y) 1_{F}(y)=\xi_{L}^{m}(d x) \hat{\mathbb{P}}^{x}, \quad X_{T} \in d y, T<\infty,
$$

事实上这是 $F$ 上的 Feller 测度 ${ }^{[6]}$.

为计算 killing 测度, 设 $\tilde{\zeta}$ 为 $\tilde{X}$ 的生命时间, $f \geqslant 0$ 是离开 $F$ 即消失的可测函数. 系统 $(\tilde{N}, \tilde{K})$,

$$
\mathbb{E}^{x}\left(e^{-\tilde{\zeta}} f\left(\tilde{X}_{\tilde{\zeta}_{-}}\right)\right)=\mathbb{E}^{x}\left(\int_{0}^{\infty} e^{-s} f\left(\tilde{X}_{s}\right) U\left(\tilde{X}_{s}, \partial\right) d \tilde{L}_{s}\right),
$$

由定理 2.2 , 有

$$
\tilde{k}(d x)=f(x) U(x, \partial) \tilde{\xi}_{\tilde{L}}^{\tilde{m}}(d x)=U(x, \partial) \xi_{L}^{m}(d x) .
$$

而 $X_{T}=\partial$ 当且尽当 $T=\infty$, 所以

$$
U(x, \partial)=\hat{\mathbb{P}}^{x}(\{T=\infty\})=\hat{\mathbb{P}}^{x}(\{T=\infty, \zeta>0\})+\hat{\mathbb{P}}^{x}(\{\zeta=0\}) .
$$

设

$$
V(d x):=\hat{\mathbb{P}}^{x}(\{T=\infty, \zeta>0\}) \xi_{L}^{m}(d x),
$$

根据文献 [6] 中定理 5.6 的证明, 另一个部分 $\hat{\mathbb{P}}^{x}(\{\zeta=0\}) \xi_{L}^{m}(d x)$ 等于 $k_{F}$, 即 $k$ 在 $F$ 上的限 制. 我们可以如下表示时间变换过程的跳跃测度和 killing 测度. 该结论的前提与文献 [6] 中的 假设不同:

\section{定理 3.2}

$$
\tilde{J}=J_{F}+U, \quad \tilde{k}=k_{F}+V .
$$

虽然时间变换过程的 Lévy 系统依赖于做时间变换加泛函的选取, 但是跳跃测度 $\tilde{J}$ 与 killing 测度 $\tilde{k}$ 与加泛函的选取是无关的, 二者均以 $F$ 为它们的支撑集.

\section{参 考文 献}


3 Douglas J. Solution of the problem of Plateau. Trans Amer Math Soc, 33(1): 263-321 (1931)

4 Doob J L. Boundary properties of functions with finite Dirichlet integrals. Ann Inst Fourier, 12: 573-621 (1962)

5 Fukushima M. On Feller's kernel and the Dirichlet norm. Nagoya Math J, 24: 167-175 (1964)

6 Chen Z, Fukushima M, Ying J. Entrance law, exit system and Lévy system of time changed processes. Illinois J Math, 50(2): 269-312 (2006)

7 Fukushima M, He P, Ying J. Feller measure and time change. Ann Probab, 32(4): 3138-3166 (2004)

8 Getoor R K. Excessive measures. Boston-Basel-Berlin: Birkhauser, 1990

9 Ying J. Revuz measures and the related formulas. Potential Anal, 8(1): 1-19 (1998)

10 Maisonneuve B. Exit system. Ann Probab, 3(3): 395-411 (1975)

11 Getoor R K, Sharpe M J. Last exit decompositions and distributions. Indiana Univ Math J, 23: 377-404 (1973)

12 Motoo M. Applications of additive functionals to the boundary problem of Markov processes. In: Proc 5th Berkeley Symp Math Stat Prob Vol 2, II, 75-110

13 Blumenthal R M. Excursions of Markov Processes. Boston-Basel-Berlin: Birkhauser, 1992

14 Motoo M, Application of additive functionals to the boundary problem of Markov processes (Lévy system of U-processes). In: Proc Fifth Berkeley Symp Math Statist Prob III. San Diego: University of California Press, 1965, 75-110 